\title{
Identification of vibration properties of a mid- rise wooden building using subspace identification method
}

\author{
Yuji Miyazu, ${ }^{1, *}$ and Takuro Mori ${ }^{1}$ \\ ${ }^{1}$ Hiroshima University, Graduate School of Engineering, Higashi-Hiroshima, Japan
}

\begin{abstract}
Recent years, the mid- and high-rise wooden building using cross-laminated timber (CLT) is on the increase in the world. In the regions highly subjected to seismic events, it is important to know the basic property of the vibration of the building to design the building to be safety enough against the seismic load; however, there is enough such data of full scale wooden buildings. In this paper, the natural period, the damping ratio, and the mode shape of a full-scale five-story wooden building are evaluated using subspace identification method. The results show that the damping ratios of higher modes tend to be lower than that of the first mode, and the natural period and the damping ratio show amplitude dependency even in the range of low amplitude.
\end{abstract}

\section{Introduction}

Recent years, mid- and high-rise wooden buildings are constructed using cross-laminated timber (CLT) in the world. Also in Japan, since the building code for CLT buildings is announced officially in 2016, it is expected that wooden tall buildings increase in the future.

When we conduct structural and seismic design of buildings, we need the basic vibration properties such as the natural period and the damping ratio. It is also important to know the variation of vibration properties during strong ground motion because wooden buildings generally show strong non-linearity under seismic load. In the research by Reynolds et al. [1], vibration properties of 11 multi-story timber buildings are investigated using ambient vibration methods; however, the investigation of vibration properties of tall wooden buildings under seismic excitation is not enough. In this paper, the vibration properties of a mid-rise wooden building using CLT, which was used as a test frame for the full-scale shake table test at E-defense [2, 3], are identified using PI-MOESP method which is one of the standard subspace identification methods.

First, we present the overview of the full-scale shake table test of the five-story CLT building, and then explain the system identification method used in this study and the identification method of the vibration properties. Finally, we provide the results obtained through white noise shaking and earthquake shaking, respectively.

\footnotetext{
* Corresponding author: yuji.miyazu@gmail.com
} 


\section{Overview of shake table test}

Figure 1 shows the five-story wooden building mounted on E-Defense, which is in Miki city in Japan. The test frame consists of CLT walls with $150 \mathrm{~mm}$ thick and CLT floors with $210 \mathrm{~mm}$ thick. The walls and the floors are connected mainly by steel plates with $4.5 \mathrm{~mm}$ thickness, screws of STS-65C, and bolts of M24 and M16. The weight of the second floor, the third to the fifth floors, and the roof are $660.0,623.1$, and $311.0 \mathrm{kN}$, respectively. Table 1 shows the information of input ground accelerations. Transverse, Longitudinal, and Vertical directions are defined in Fig. 1 by yellow arrows. Simulated earthquake 1 and 2 are the middle- and the large-scale earthquake ground motions defined in the Japanese Building Standards Act, respectively, and JMA Kobe is the ground motion recorded during the 1995 Kobe earthquake. In Fig. 2, the location of acceleration sensors, the data of which are used in the system identification described in the next section, are indicated by black square, and the blue and the red arrows shows the measurement direction of each sensor.

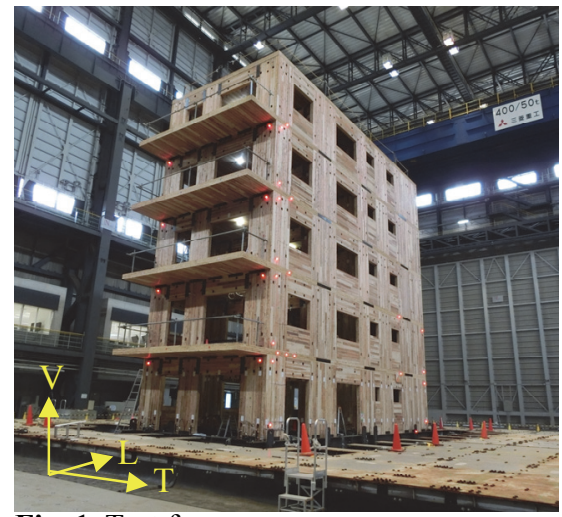

Fig. 1. Test frame.

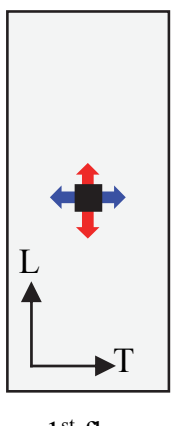

$1^{\text {st }}$ floor
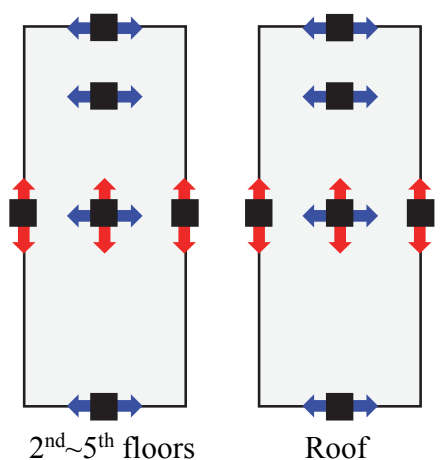

Fig. 2. Location of acceleration sensors.

Table 1. Input ground accelerations

\begin{tabular}{cccc}
\hline No. & Name & Direction & Max. acceleration $\left(\mathrm{cm} / \mathrm{s}^{2}\right)$ \\
\hline A-02 & White noise & $\mathrm{T}, \mathrm{L}$ & $\mathrm{T}=21, \mathrm{~L}=44$ \\
A-03 & Simulated earthquake 1 & $\mathrm{~L}$ & 145 \\
A-04 & Simulated earthquake 1 & $\mathrm{~T}$ & 115 \\
A-05 & White noise & $\mathrm{T}, \mathrm{L}$ & $\mathrm{T}=23, \mathrm{~L}=46$ \\
A-06 & White noise & $\mathrm{T}, \mathrm{L}$ & $\mathrm{T}=21, \mathrm{~L}=43$ \\
A-07 & Simulated earthquake 2 & $\mathrm{~L}$ & 798 \\
A-08 & White noise & $\mathrm{T}, \mathrm{L}$ & $\mathrm{T}=20, \mathrm{~L}=45$ \\
A-09 & White noise & $\mathrm{T}, \mathrm{L}$ & $\mathrm{T}=22, \mathrm{~L}=54$ \\
A-10 & Simulated earthquake 2 & $\mathrm{~T}$ & 622 \\
A-11 & White noise & $\mathrm{T}, \mathrm{L}$ & $\mathrm{T}=21, \mathrm{~L}=82$ \\
A-12 & White noise & $\mathrm{T}, \mathrm{L}$ & $\mathrm{T}=20, \mathrm{~L}=64$ \\
A-13 & JMA Kobe & $\mathrm{T}, \mathrm{L}, \mathrm{V}$ & $\mathrm{T}=805, \mathrm{~L}=1014, \mathrm{~V}=389$ \\
A-14 & White noise & $\mathrm{T}, \mathrm{L}$ & $\mathrm{T}=19, \mathrm{~L}=54$ \\
※ $=$ Transverse, L=Longitudinal, V=Vertical &
\end{tabular}

\section{Identification method of vibration property}

In this paper, the relation between the input ground acceleration and the floor response acceleration is expressed by the state-space model as follows:

$$
\begin{aligned}
x(k+1) & =A x(k)+B u(k) \\
y(k) & =C x(k)+D u(k)+v(k)
\end{aligned}
$$


where $u(k), y(k)$, and $v(k)$ are vectors of the input ground acceleration, the floor response acceleration, and a colored measurement noise, respectively. $A, B, C$, and $D$ are system matrix, and they are identified by PI-MOESP method proposed by Verhaegen $[4,5]$.

Using the eigen value $p_{i}$ and the eigen vector $\psi_{i}$ of the matrix $A$, the natural period $T_{i}$, the damping ratio $h_{i}$, and the mode $u_{i}$ are calculated by Eq. (2a-c), respectively. $\Delta t$ is the sampling time of acceleration data.

$$
T_{i}=\frac{2 \pi \Delta t}{\left|\log p_{i}\right|}, \quad h_{i}=\frac{-\log \left|p_{i}\right|}{\left|\log p_{i}\right|}, \quad u_{i}=C \psi_{i}
$$

\section{Results}

In the first part of this section, the natural period, the damping ratio, and the mode of the test frame subjected to white noise are identified, and then the relation between these values and the experienced damage of the test frame is examined. In the second part, the time history of the natural period and the damping ratio under earthquake shaking are evaluated, and then the amplitude dependency of these values is investigated.

\subsection{White noise shaking}

Using subspace identification methods, the identification result highly depends on the model order $N$ and the number of block rows of block Hankel matrix $s$. In this paper, the stabilization diagram in which $N$ and $s$ are selected as parameters is described at first to estimate visually the proper values of $N$ and $s$, and then the natural period, the damping ratio, and the mode which satisfy criteria defined later are selected as a reliable value.

Figure 3 shows the stabilization diagram obtained by A- 02 test. The area of each circle plot represents the magnitude of damping ratio. It is clearly seen that the natural frequency and the damping ratio become stable with increase of the value of $s$. In this paper, the criteria to judge the reliability of the identified values are as follows:

$$
\begin{gathered}
\left|\frac{f_{i}-f_{i+1}}{f_{i}}\right| \leq \varepsilon_{f}=1 \%, \quad\left|\frac{h_{i}-h_{i+1}}{h_{i}}\right| \leq \varepsilon_{h}=10 \% \\
\operatorname{MAC}\left(\varphi_{i}, \varphi_{i+1}\right)=\frac{\left|\varphi_{i}^{*} \cdot \varphi_{i+1}\right|^{2}}{\varphi_{i}^{*} \cdot \varphi_{i} \cdot \varphi_{i+1}^{*} \cdot \varphi_{i+1}} \geq \operatorname{MAC}_{\varphi}=98 \%
\end{gathered}
$$

where $f_{i}$ and $h_{i}$ are the natural frequency and the damping ratio of $i$ order. MAC is the modal assurance criterion calculated by the mode of $i$ and $i+1$ order, $\varphi_{i}$ and $\varphi_{i+1}$. Superscript $\left.{ }^{*}\right)$ in Eq. (3c) denotes complex conjugate transpose.

Figure 4 shows the mode, the natural period, and the damping ratio from the first to the fourth modes which are indicated by $\boldsymbol{\nabla}$ in Fig. 3. It is seen that all the mode in the transverse direction include torsional motion. The damping ratio of the first mode is nearly $3 \%$, and those of higher modes are lower than the first mode damping ratio except for the value of the second mode in the transverse direction. This result indicates that the stiffness proportional damping, which is often used as the model of the modal damping in time history response analysis, overestimates the damping ratio of the higher modes.

In Figs. 5 and 6, the relation of the natural period or the damping ratio and the maximum story drift experienced by earthquake shakings are indicated, respectively. The 
vertical axis in Fig. 5 is the natural period ratio, which is defined as the ratio of the natural period $T$ to that of the initial value $T_{\text {initial }}$ obtained by A- 02 test. It is found that the natural period and the damping ratio increase with increase of the experienced maximum story drift, and basically, the trend is noticeable in the lower mode.

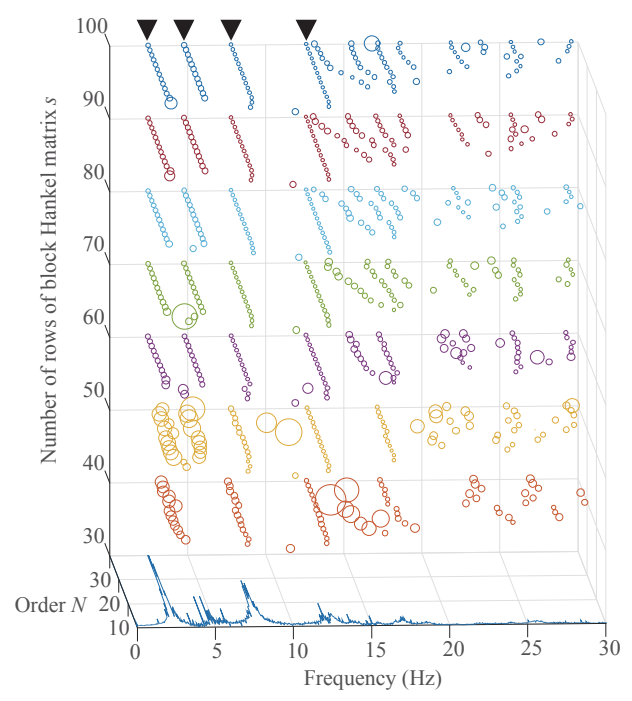

(a) Transverse direction

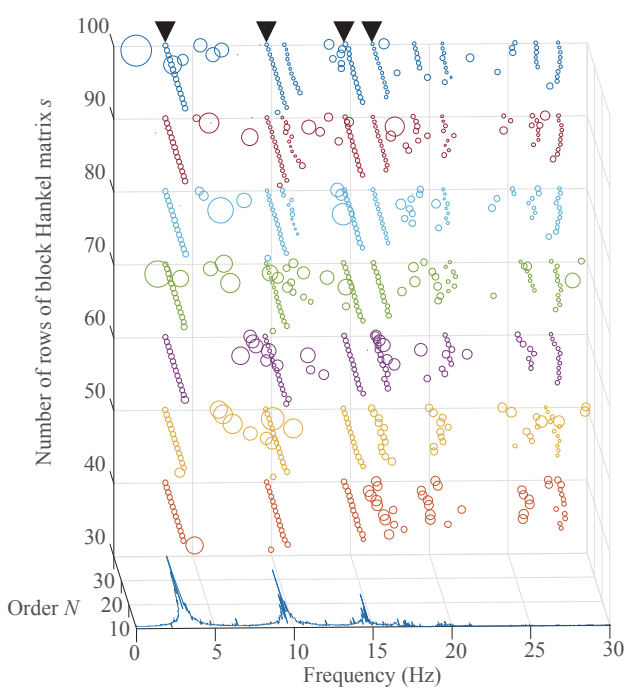

(b) Longitudinal direction

Fig. 3. Stabilization diagram obtained by A-02 test.

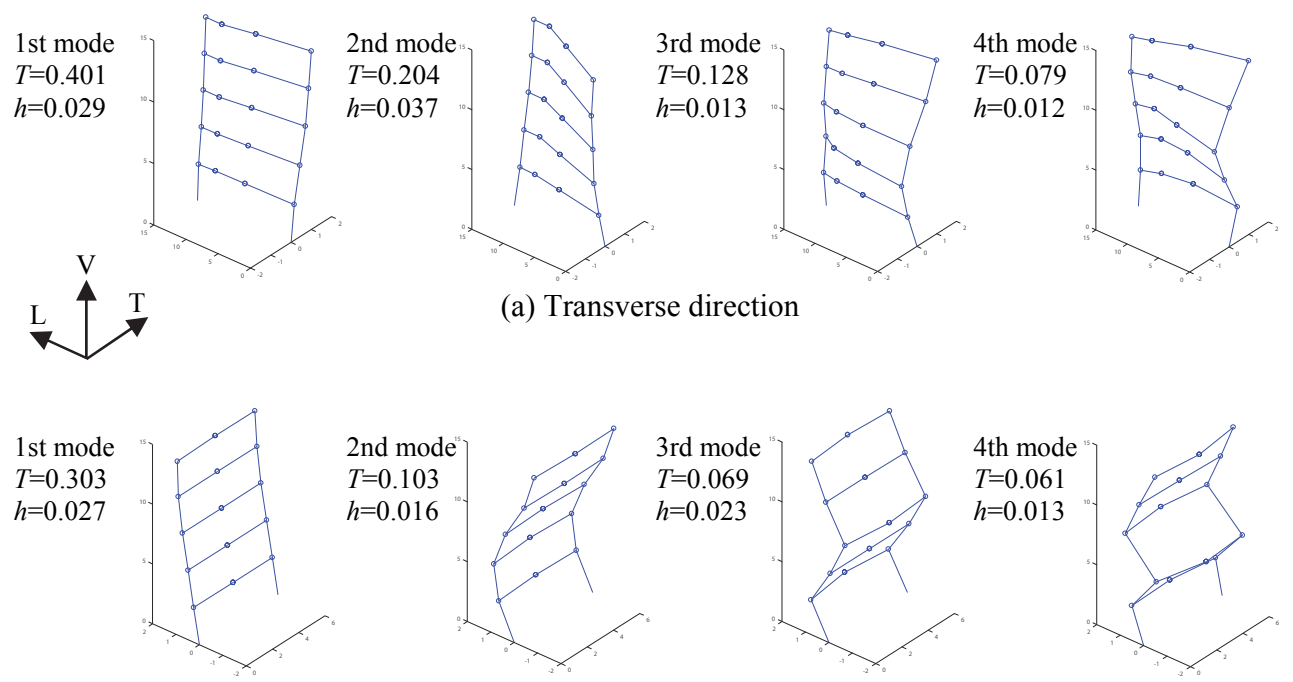

(b) Longitudinal direction

Fig. 4. Mode shape obtained by A-02 shaking. 


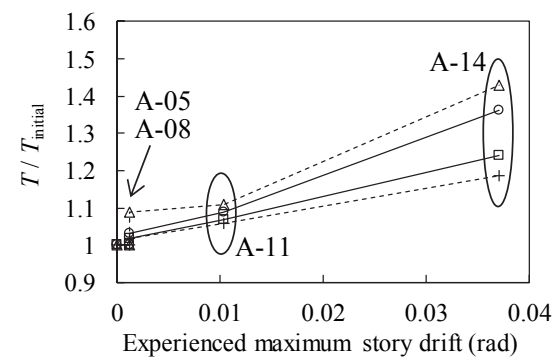

(a) Transverse direction

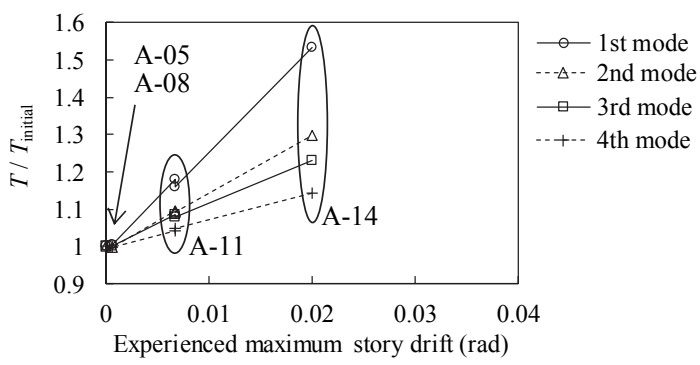

(b) Longitudinal direction

Fig. 5. Relation between experienced maximum story drift and natural period ratio.

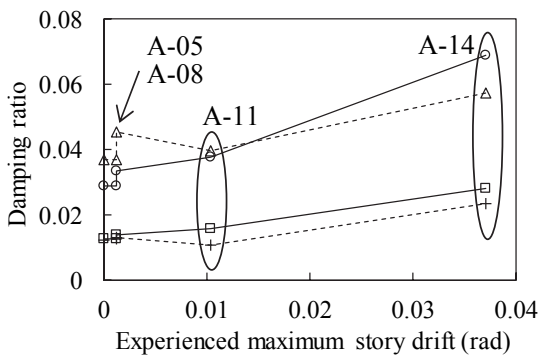

(a) Transverse direction

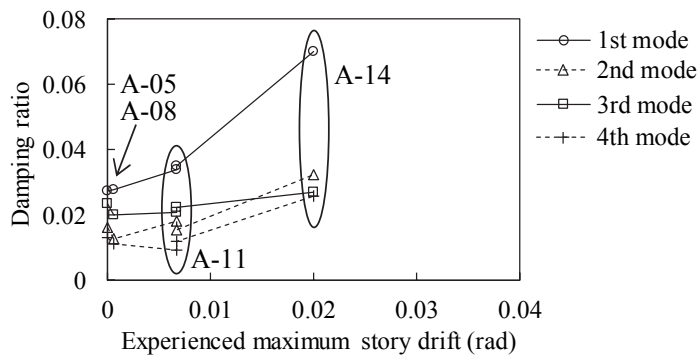

(b) Longitudinal direction

Fig. 6. Relation between experienced maximum story drift and damping ratio.

\subsection{Earthquake shaking}

When we process the data obtained by earthquake shakings, in order to investigate the variation of vibration properties due to nonlinear behaviour, the subspace identification is conducted every second using the data during 5 seconds. In this section, acceleration sensors located at the centre of the each floor and the roof are used for the identification.
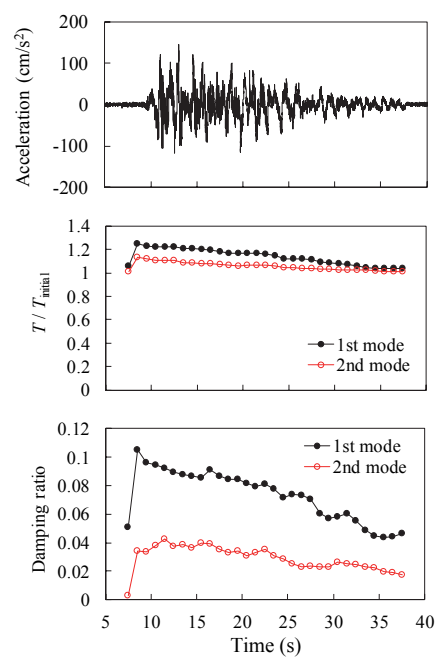

(a) A-03
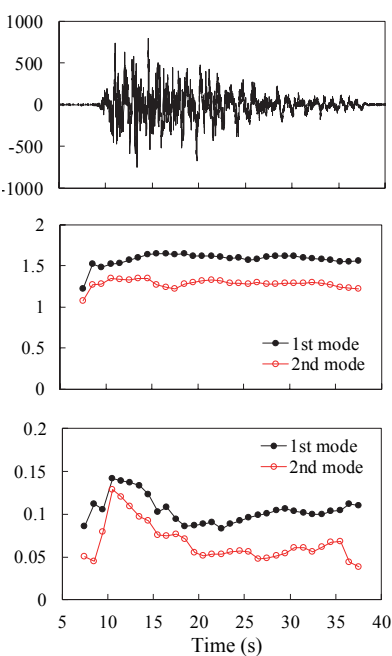

(b) A-07
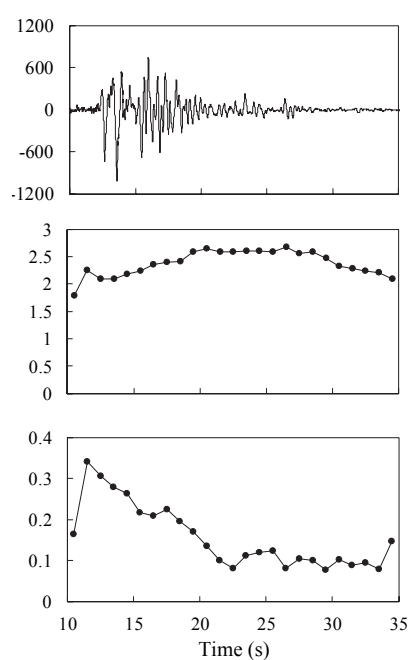

(c) A-13

Fig. 7. Time history of input acceleration, natural period ratio, and damping ratio. 
Figure 7 shows the time history of the input ground motion, the natural period ratio, and the damping ratio in the longitudinal direction during A-03, A-07, and A-14 tests. In the tests of A-03 and A-07, the values of the first and the second modes are identified. It is clearly seen that the natural period and the damping ratio become larger in all the modes during ground motions, especially in A-14 test, the damping ratio reaches nearly $35 \%$ and the natural period becomes 2.5 time longer than the initial period.

In order to investigate the amplitude dependency of the vibration properties, using the data of Fig. 7(a), relations between maximum story drift and the natural period ratio or the damping ratio are shown in Figs. 8(a) and (b), respectively. The story drift used here is the ratio of the roof displacement to the height of the building, and the roof displacement is calculated by integration of the roof acceleration applied high-pass filter of $0.1 \mathrm{~Hz}$. In both figures, the amplitude dependency is clearly shown in both the natural period and the damping ratio.

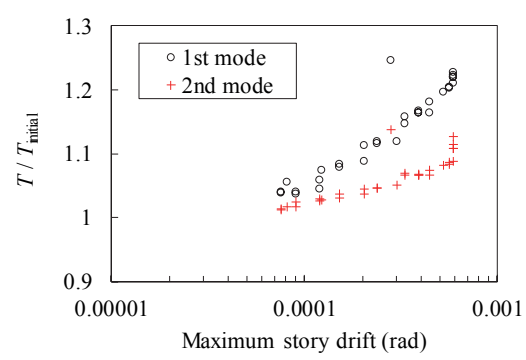

(a) Natural period ratio

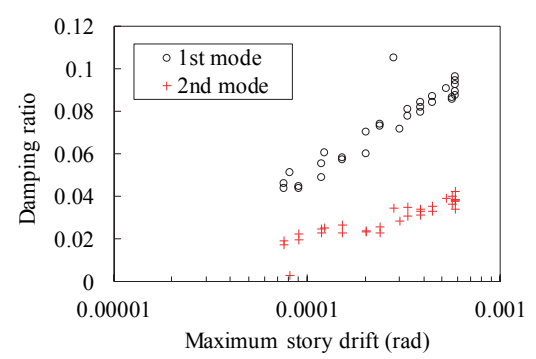

(b) Damping ratio

Fig. 8. Amplitude dependency of natural period and damping ratio.

\section{Conclusion}

In this paper, the vibration properties of the five-story CLT building are identified by the subspace identification method. Through the white noise shakings of the building, it is found that the damping ratio of the higher modes are not large compared with that of the fist mode. From the result of the earthquake shakings, it is clearly shown that the natural period and the damping ratio have amplitude dependency even in the range of low amplitude.

In this paper, the experimental data obtained by ASEBI, which is operated by National Research Institute for Earth Science and Disaster Resilience (NIED), is used.

\section{References}

[1] T. Reynolds et al., INTER, Meeting 49, pp. 365-377 (2016)

[2] National Research Institute for Earth Science and Disaster Resilience (NIED), Experiment for Verification of Structural Performance of CLT Buildings, https://www.edgrid.jp/datas (2016)

[3] N. Kawai, et al., WCTE2016, MS4-07B (2016)

[4] M. Verhaegen, Int. J. Control 58, pp. 555-586 (1993)

[5] M. Verhaegen, V. Verdult. Filtering and System Identification (2007) 